\title{
A User-Centered Approach for Cloud Service Selection and Recommendation
}

\author{
Aqsa Ashraf ${ }^{1}$, Imran M. Rabbani ${ }^{1}$, Ana Maria Martinez-Enriquez ${ }^{2}$, \\ Aslam Muhammad ${ }^{1}$ \\ ${ }^{1}$ Department of CS \& E, University of Engineering \& Technology, \\ Lahore, Pakistan \\ ${ }^{2}$ Department of CS, CINVESTAV-IPN, Ciudad de Mexico, \\ Mexico \\ ammartin@cinvestav.mx, aqsa.ashraf4@gmail.com, \\ imranmrabbani@gmail.com, maslam@uet.edu.pk
}

\begin{abstract}
Cloud computing is one of the most emerging industries and offers scalable computing resources and on-demand packages. A large number of cloud services are being offered by many service providers, a vendor supplies many services or same service is provided by many vendors, which creates the selection issue of appropriate cloud service from a big pool. Quality of service plays important role for better service choice. For this reason, cloud service recommendation systems are proposed in the literature that makes it easy for a user to decide which service to choose. However, user needs and requirements like economy, feedback, etc. are least considered, In our approach, an efficient cloud service recommendation system has been developed enabling users to hire a cloud service according to their own requirements. The aim is to test services according to user needs and some stated parameters. Users select the criteria from given parameter repository, the quality attributes like security, privacy, data storage, etc. and thus services are evaluated. Finally, results are shown providing user with the flexibility and opportunity to select best cloud service.
\end{abstract}

Keywords: Cloud computing, recommendation system, quality attributes, user feedback.

\section{Introduction}

Information technology has become very popular among organizations by providing them to communicate and create more efficiently and reliably [1]. Cloud computing is making its place in this area. It is a model for enabling convenient, on-demand network access to a shared pool of configurable computing resources (networks, servers, storage, applications, and services) that can be rapidly provisioned and released with minimal management effort or service provider interaction [2][3]. Instead of keeping data in our own hard drive or updating applications for our needs, we use a service over the Internet, at some other location, to store our information or use its applications. 
Main focus of a cloud is to provide reliable, secure, sustainable, fault tolerant data sharing through web based applications [4]. Several well-known and huge business enterprises like Google, Amazon and IBM are offering different cloud services to users now [5][6].

The infrastructure of cloud computing allows more efficient use of hardware and software. Cloud service selection is different from web service selection in terms of quality of service and Service Level Agreement [7]. There exist challenges from service adoption perspective. The basic issue of cloud computing is to provide reliable services and allow consumers to access applications and data on demand. Different vendors follow different criteria and fulfil certain demands. Any cloud service offered by a service vendor is different in various perspectives and a description of these characteristics is as follows [8]:

On-demand service: Allows the user to unilaterally provision the computing resources required for that particular service without human interaction. Cloud providers automatically handle the user request for extra storage or computing time. The advantage of provisioning is that it allows the cloud to manage its resources on demand basis and allows it to keep the security, availability and functionality of a service at high level.

Network access: Services are available to user and are accessed through the internet or devices that are connected or networked. There is another scenario in which a software component is installed on the consumer device in order to use the service but somehow this approach is a least favorite to users due to the reason of a third party component installation as it puts an organization's security and trust on risk.

Rapid Elasticity: Certain capabilities to use a service are provided to the user which may sometime seem as unlimited to user so these capabilities at any time can be appropriated in any quantity.

Measured Service: In case of occurrence of an issue during the provision of services, it is supervised to get resolved and the services are monitored and measured for billing according to their utilization. The service monitoring helps in recovering from a fetal error in the production environment and for system crashes as well. Logging history is also maintained with service monitoring and this history is used as an archive in order to debug a service if any problem arises in service functionality.

Resource Pooling: The computing resources of a service provider are pooled so that it can serve multiple service users by using a multi-tenant model. These virtual and physical resources are assigned and reassigned dynamically to the user according to their demand.

A thorough introduction of service models given by cloud is as follows [9][10][11]:

Software as a Service (SaaS) provides users to connect with the service providers to use the application, but they do not control the infrastructure, operating system or network infrastructure. There is no need for user to have heavy resources with installed application software. Users only need to have browsers on their devices to connect to internet in order to use the application in cloud. A daily, weekly, monthly and annually subscription period is provided and the end user is charged according to that duration working on "pay-as-you-go" mechanism [12]. An example of SaaS would be Google docs. A Cloud Service Broker helps finding suitable SaaS services [13].

Platform as a Service (PaaS) provides users to utilize all facilities on the cloud to develop and deliver their Web application and services to the end users. This service 
may include development, testing or the storage resources to complete the life cycle of services. Some examples of PaaS are Microsoft's Azure, Google App Engine, Engine Yard etc. A framework for user management, resource management and access management in PaaS is maintained [14].

Infrastructure as a Service (IaaS) provides computing infrastructure and resources like firewalls, image library file based storage and load balancers. Users can use the visualization resources as a fundamental infrastructure for their applications. User has access to computational resources and these resources may be a CPU, network or storage [4]. Some of the known IaaS vendors are Amazon AWS, Go Grid, etc.

DataCenter as a Service (DCaaS) offers all the features of IaaS plus some additional features such as providing the infrastructure ownership to user are included in this model. Major cloud vendors of DCaaS model are Rackspace, GoGrid, Amazon AWS and business entities usually use services by these vendors if there is need to have wide control over equipment.

A large number of cloud services are being available on internet with time. Some of the examples are network and storage services provided by different vendors. The important point is to make a decision in selecting the best suited service when there are multiple service providers. Problem arises when a user cannot decide which service to select when that particular service is being provided by multiple vendors. Internet community lacks information about various services, their performance and other quality attributes. A service provided by different vendors would have different quality attributes so the user might end up selecting a service that is not quite up to the mark and does not nearly fulfil user's requirements. So, a system is developed that is easy to use and provides users with best results to their requirements.

The related work is given in Section 2, followed by the proposed system in Section 3. A case study is discussed in Section 4 and conclusions are presented in Section 5.

\section{Related Work}

In the cloud recommender in [15][16], a repository is maintained in which infrastructure services are stored from different providers. Domain knowledge of multiple configurations of infrastructure services is identified and formalized. This knowledge is then implemented in a recommender service on top of a relational data model. It implements transactional execution procedures and applies well defined SQL semantics for query, insert and delete infrastructure services configuration.

The algorithm proposed in paper [13] gives ranking of SaaS providers according to their offerings matching the consumer requirements. A CS Recommender in [17] consists of five main components as crawler, cloud service identifier, indexer, search engine and recommender system. Recommender uses both collaborative and content based approaches by calculating an average. System starts with the content based recommendation results and then shifts to collaborative results when number of user increases. Approach in [18] uses the data mining technique K-means clustering for service recommendation which provides computational simplicity.

A Service Measurement Index (SMI) framework given in [19, 20] was designed for the comparison and ranking of cloud services. Cloud customers can also use these indexes to compare different cloud services. Pseudo ratings are constructed based on 
implicit feedback in [21]. It implements matrix factorization. Ratings are extracted from user watch list. Model is retrained for new user. Ratings are gathered into a dataset and a strategy is applied to recommend a service to user. Online pattern is used for new user ratings and the model is retrained according to the new feedback for future recommendations.

Service selection and recommendation are supported by these solutions but not all the systems take into account the feedback provided by previous users of a particular service and most importantly the requirements of a user regarding service quality attributes. Moreover, user needs are approximated and not thoroughly looked into. The proposed system makes service selection easy for cloud users by evaluating services and leaving the choice of quality parameters totally on service user.

\section{Cloud Service Recommender System}

The proposed solution, illustrated in Figure 1 has been developed and divided into separate components and these are done using software approach. The service recommendation task has been accomplished in a simple way by the system gathering data, implementing existing technologies, collecting users, taking comments in feedback form and maintaining quality attributes. Along with the collection of data, the conditions or constraints for criteria were also collected from service seeker or user which is a very important step that should be carried out during service search. This makes the commencement of a very first step as data collection which is the most important information as the service evaluation is carried out on this information basis.

The core element of CSRS is the repository which is a collection of various quality attributes. Due to the rapid increase in the number of web services it has become very important to build a service which is trustworthy and for this reason, QoS attributes have become an important factor [22]. A large number of service attributes are available and different sets of attributes have been developed [23]. Some of the quality attributes supported by this system are Stability, Cost, Security, Availability, Performance and services are thoroughly checked and evaluated by the system. Services are analysed and the analysis algorithm is performed on services according to the selection of quality of service attributes. All the related parameters and features of services and characteristics of cloud service providers are gathered and the attributes are selected and saved for the specific cloud services selected for evaluation. After the gathering and saving of data, pre-processing steps are taken such as filling some missing information, incorrect or duplicate information removal from the data collected.

Vendors are maintained along with their services on a page and links are given for each service of a service provider. User is then provided with the option of selecting any vendor and its service from vendor service list or can seek the help of cloud recommender system by selecting the desired parameters and let the system evaluate all the services of all the vendors available and this evaluation is done on the basis of selected parameters by the user.

In order to collect user reviews or feedback according to their service usage, users are presented with a survey to input their response for a service based on the attributes stated in the survey form. Major module is the quality of service attribute rating collection in the form of feedback for each attribute of a service and weightage 
associated with each attribute is also collected. All of this collected information is then passed onto the database created for storing user feedback. As services are evaluated based on the review or feedback of user on its features, properties or attributes so ranking of each attribute is collected from user review. Another major module is the dashboard, where all the available services are shown to the user along with each service description, its service type whether it is storage, computing or security service and its vendor information. User can navigate to individual and separate pages for every service and can view its details and also provide feedback for service. The first module is the graphical user interface where users are given the option whether to choose any service by themselves or direct to system repository and select the desired parameters and let the system do the calculation.

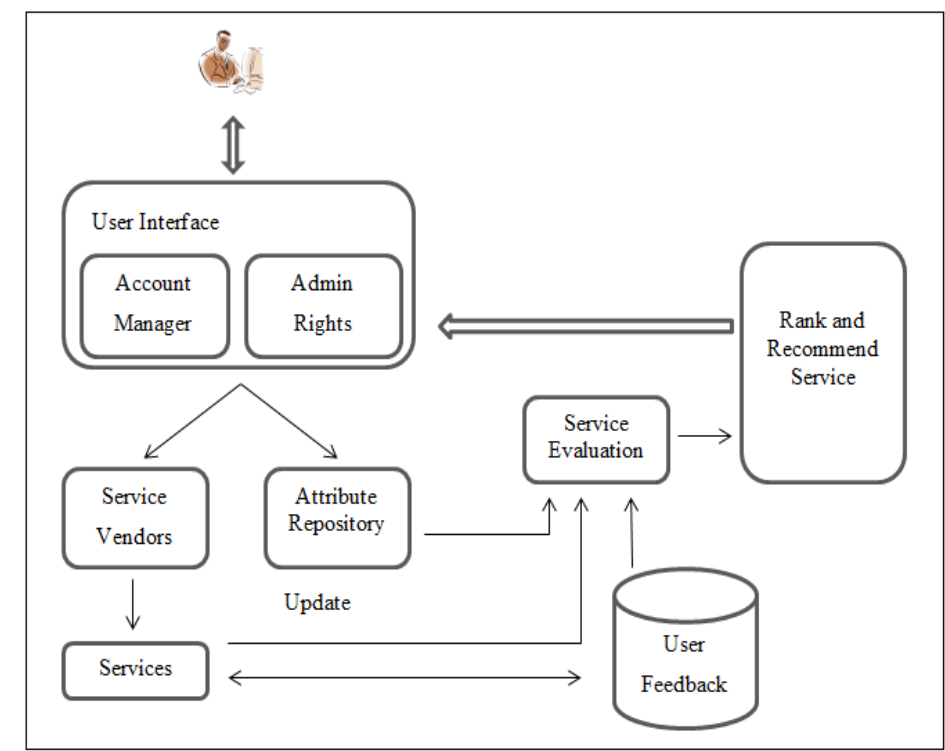

Fig. 1. Architecture of Recommender System.

The QoS parameters selected by user are passed on further to give query results for specified criteria. This modular approach of the system offers user to work in a number of phases and makes selection easy for user for best service based on user criteria. Performance of a cloud service provider depends on the performance of all its services provided. Performance of a service provider is calculated using Eq. 1.

$$
P_{s p}=\sum_{m=1}^{n}\left(p_{m}(s)\right),
$$

where $n$ is the total number of services of cloud service provider supported by the system. This $p_{m}(s)$ is the score of a service based on the quality attributes selected from repository given in Eq. 2. 


$$
p_{m}(s)=\sum_{i=1}^{R_{U}}\left(f_{i}-t_{i}\right), \quad R_{U} \subseteq R
$$

Where $R$ represents the attribute repository which contains different quality of service parameters and $R_{U}$ is the number of user stated or user selected quality parameters which is clearly a subset of the original parameter repository as shown above. $f_{i}$ is the user feedback with respect to attribute $i$ and $t_{i}$ is the company value for the attribute or quality parameter $i$ as a threshold value. Total feedback against a single service is calculated by using Eq. 3:

$$
\begin{aligned}
& f_{\text {total }}=\frac{f_{1}+f_{2}+\cdots+f_{n}}{n}, \\
& 0<f_{\text {total }}<10,0<t_{i}<10,
\end{aligned}
$$

where $n$ is the total number of service users.

This module functions according to the results collected from users in the form of feedback and the threshold value assigned to the services. The system is implemented using these algorithms on asp.net software in the form of a web solution. When a service is rated by a user, the ratings for all its attributes along with user information are stored in database. End user is first registered with the system and after registration assigned an account. On signing in, user is given the option of selecting services by themselves or move to the system repository.

In system repository, quality parameters are maintained and users are asked to select the desired parameters on which they want services to be evaluated and recommended. On selecting parameters by users, the evaluation processing of services starts which takes user feedback and company value as inputs and after processing according to the designed algorithm, produces some results which evaluates all services based on the parameter selection accordingly. According to the results presented, user can choose first service as it is ranked as the best among all others based on user criteria. Working of the recommender system is illustrated in Figure 2.

\section{Finding Service Rank}

1. Login profile

2. ServiceList[]

3. Select Service Type $\mathrm{p}$

4. Select $\mathrm{n}$ parameter/parameters from $\mathrm{R}$

5. Services $\leftarrow$ Apply algorithm with $\mathrm{f}$ and $\mathrm{t}$

6. Get MAX //Load Service

7. If(Service $\mathrm{k}=\mathrm{MAX})$

8. Set Service k rank $\rightarrow 1$

9. $\quad$ Else if(Service $\mathrm{k}=\mathrm{MAX}-1)$

10. Set Service k rank $\rightarrow 2$

11. Output SortedServiceList[] 


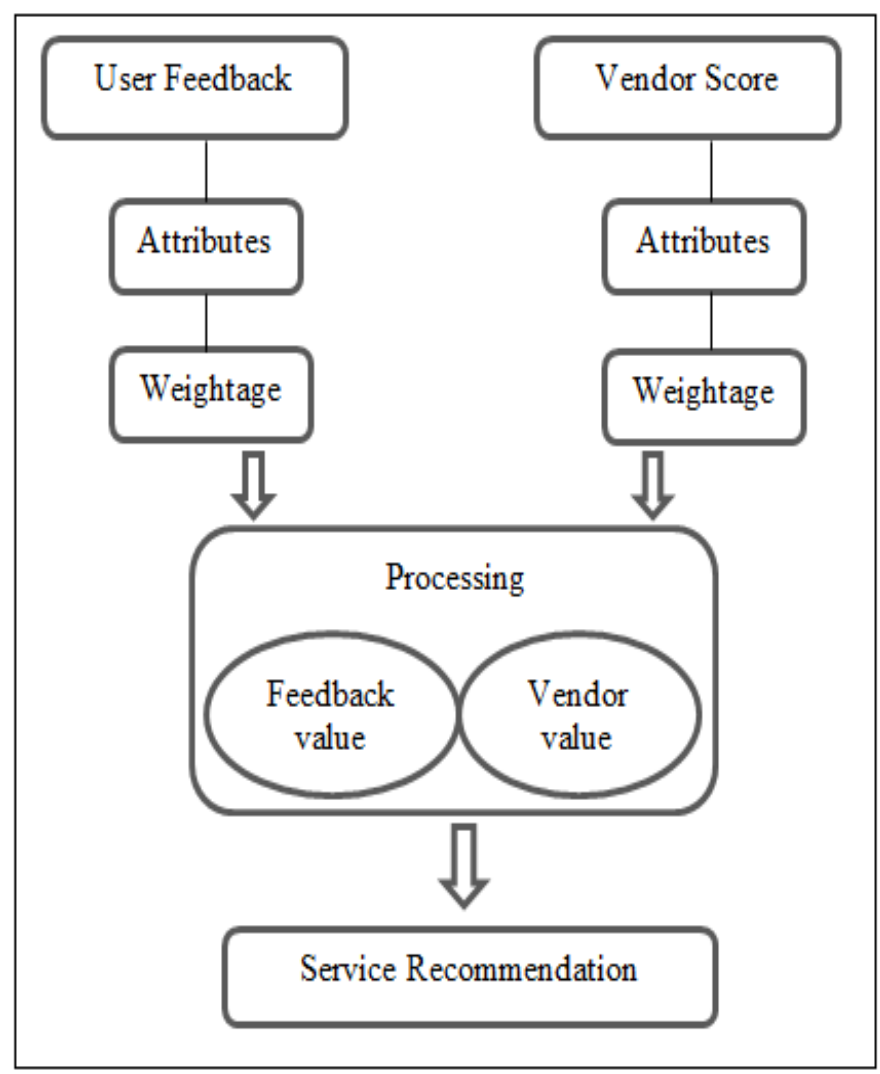

Fig. 2. Recommender System Working.

This pseudocode shows the initial step of user login and then selecting the type of service needed to be evaluated and recommended. The service type which is used as a case study is storage services. One or more than one parameters are selected from repository and algorithm is applied on all the services of given type using feedback and threshold values. Service with maximum value(MAX) obtained is ranked highest among the others and is recommended as the best suited service according to user's quality requirements.

\section{Storage Service Recommendation}

The services evaluated by the recommender system are given in Table 1 . The table shows some of the cloud storage services provided by different vendors over the internet. The need for storage service arises for several reasons such as if the user device space is not enough to keep large amount of data or organizations have the need to keep data on cloud. The user data is stored on a space provided and managed at the server side and only users with registered accounts have access to their own data. 
Table 1. Cloud Services with Type.

\begin{tabular}{|c|c|}
\hline Service Name & Service Type \\
\hline GoogleDrive & Storage \\
\hline OneDrive & Storage \\
\hline IDrive & Storage \\
\hline DropBox & Storage \\
\hline SugarSync & Storage \\
\hline JustCloud & Storage \\
\hline BackBlaze & Storage \\
\hline CrashPlan & Storage \\
\hline
\end{tabular}

An introduction to the services shown in the Table I is given as follows.

- GoogleDrive provides a free storage of $15 \mathrm{~Gb}$ initially along with presentation builder, spreadsheet and word processor. This service also has applications for managing files and data from cellular phone. Third party applications are also supported to sign documents or send faxes etc.

- OneDrive provides free $15 \mathrm{~Gb}$ of initial storage which is further extended by completing a few offers. At present, it offers storage of $5 \mathrm{~Gb}$ to the users whereas, the Microsoft office subscribers have a storage limit in terabytes. It implements modern design of Microsoft user interface. There is an option of creating files and folders on the web using the Microsoft office online platform. Social networks could be linked to the storage account which allows file sharing and permissions are set for the other users to edit the data.

- IDrive provides $5 \mathrm{~Gb}$ of free storage on account registration and $1 \mathrm{~Tb}$ for personal pro level means who are the payers for this service. After account creation, a key type is selected by user whether it is a private encrypted key or IDrive managed default key. IDrive also supports social media backup and this feature lets user to back up all the data such as audio files, video files and pictures.

- DropBox provides storage service to linux and blackberry users as well and for windows it offers an official phone application as well. Upto $2 \mathrm{~Gb}$ storage is free whereas a specific amount needs to be paid in order to get more storage. Certain offers are available with which the providers provide user with free upgrades or some storage as a reward.

- SugarSync provides free storage of $5 \mathrm{~Gb}$ after creating an account which is more than the storage provided by Dropbox. But free account is provided only for 30 days after that files access or account usage is charged as per usage. Free trial period is increased if this service is recommended and referred to other users as well and the amount is $500 \mathrm{mb}$ per person and another way to increase amount is 
by completing different tasks. Data uploading speed can be managed at user end based on the internet speed of service user.

- JustCloud gives free storage of $5 \mathrm{~Gb}$ on new account registration and on recommending this service to other friends gives additional storage at the rate of $1 \mathrm{~Gb}$ per person. JustCloud servers implement AES 256 bit encryption but its security is somehow limited to just basic features. File sharing with the access limitation of view or read only and edit is available and it also supports file versioning.

Table 2. Weighted Score of Services.

\begin{tabular}{|c|c|c|c|c|c|}
\hline Service & Availability & Cost & Security & Stability & Performance \\
\hline IDrive & 6.87 & 6.62 & 6.75 & 5.5 & 6.37 \\
\hline JustCloud & 7 & 6.57 & 6.42 & 7 & 6.28 \\
\hline OneDrive & 7 & 6.57 & 6.42 & 7 & 6.28 \\
\hline SugarSync & 7 & 7.12 & 6.62 & 7.12 & 6.87 \\
\hline BackBlaze & 7 & 7.12 & 6.62 & 7.12 & 6.87 \\
\hline CrashPlan & 7.12 & 7.75 & 6.12 & 7.37 & 7.37 \\
\hline DropBox & 6.28 & 6.57 & 7 & 5.85 & 5.71 \\
\hline GoogleDrive & 6.62 & 6.87 & 6.37 & 6.37 & 5.87 \\
\hline
\end{tabular}

- BackBlaze offers an unlimited storage for about 50 to 58 dollars a year which is a very small amount compared to other backup services. The problem with this service is that it is very slow and offers a minimum amount of features which is not an attractive property for a service and the decision for backing up data is taken by the service instead of service user and there is no availability of sharing files stored on the cloud. From user level SSL/TLS encryption is done for transferring files to cloud and AES encryption is done at the service server level. A key is stored on the servers in a secure format.

- CrashPlan provides a 30 day free trial and after that pricing according to selected plan is started. There are no limits in file sizes and their storage but it does not support features like collaboration, file sharing or synchronization. The initial backup time is slow and it may take almost a day to backup all files on cloud but is a very powerful online backup solution. A peer-to-peer backup is supported which allows a user to back-up their data on someone else's computer who have also an account for CrashPlan and allow some space for their friends data and all of this is done with encryption. Level of security differs for different plans. Files are transferred using 128 bit SSL encryption.

The domain of cloud storage services is very vast and a huge number of storage and backup related services are available which cover major areas of this field. Table 2 shows the weighted score of each parameter supported in the system repository for given storage services. 


\section{All Parameter Selection}

A scenario is the selection of all quality parameters and it will be checked in this scenario that which storage service is recommended by the system. The selection is shown in Figure 3 which shows that all the parameters are selected from parameter repository page for services to be evaluated based on them and these are availability, cost, security, stability and performance.

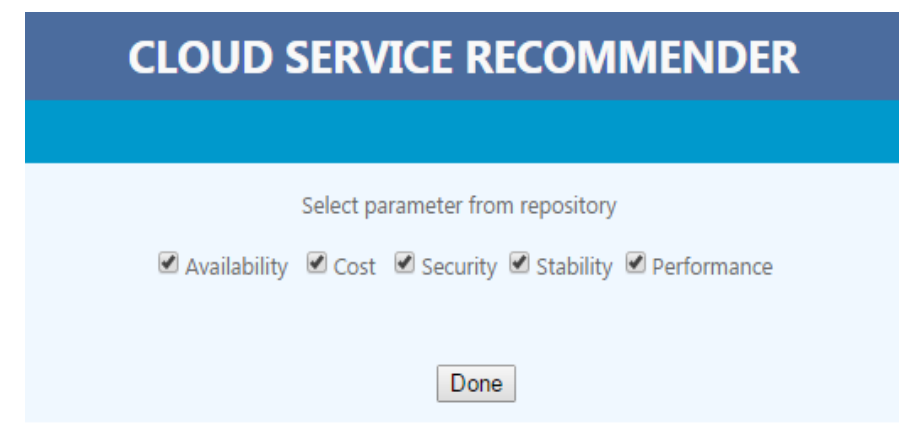

Fig. 3 All Parameters Selection.

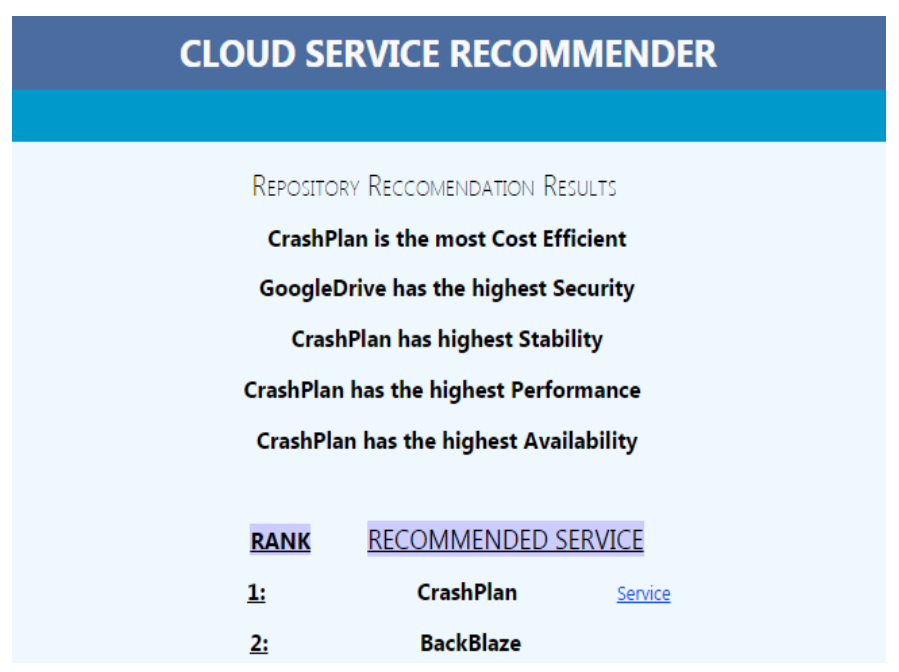

Fig. 4 Service Recommendation for All Parameters.

System now evaluates all the services according to all of the selected parameters and its result is shown in Figure 4.

Figure 4 shows that according to the selection of all the parameters, the best suited cloud storage service is CrashPlan and it is the service being recommended by the system and the second best service according to the result is Backblaze being shown at the bottom. The top of the above Figure shows results for all the parameters individually meaning that according to the system evaluations, which service falls at top for any 
individual quality parameter. This shows the fine and efficient working of the recommender system which provides detailed description of services.

\section{Conclusion}

In widely spreading and complex domain of cloud computing, it is necessary to assist cloud users in selection of services offered by this paradigm. Problem arises with multiple options which makes decision making difficult for user. Selection may be affected by multiple parameters like cost, efficiency, resource nature, satisfaction, among other. So, we develop a powerful technique to recommend cloud service to its seekers which not only considers the user requirements but also quality of service and feedback of several other clients. The service which fulfills the maximum requirements of the user is recommended consequently the surfing time is decreased and service seeker can avail the best of all available cloud services. In future, the feedbacks and opinions of users could be organized according to their dates so that it defines the concept and working of a service in a particular time.

\section{References}

1. Foster, I., Zhao, Y., Raicu, I., Lu, S.: Cloud Computing and Grid Computing 360-Degree Compared. In: Workshop on Grid Computing Environments (2008)

2. Technology, N.I.o.S.a.: The NIST Definition of Cloud Computing. Special Publication, pp. 800-145 (2011)

3. Buyya, R., Broberg, J., Goscinski, A.: Cloud Computing Principles and Paradigms (2011)

4. Neela, K.L., Kavitha, V.: A Survey on Security Issues and Vulnerabilities on Cloud Computing. Int. Journal of Computer Science and Technology (2013)

5. Kumar, S.,Versteeg, S., Buyyaa, R.: A Framework for Ranking of Cloud Computing Services. Elsevier, pp. 1012-1023 (2013)

6. Iosup, A., Ostermann, S., Yigitbasi, N., Prodan, R., Fahringer, T., Epema, D.: Performance analysis of cloud computing services for many-tasks scientific computing. IEEE Transactions on Parallel and Distributed Systems, pp. 931-945 (2011)

7. Preeti Gulia, S.S.: Dynamic Ranking and Selection of Cloud Providers Using Service Level Agreements. Int. J. of Adv. Research in Computer Science and Software Engineering (2013)

8. Talia, D.: Cloud computing and Software Agents: Towards Cloud Intelligent Services. Workshop on Objects and Agents (2011)

9. Dimpi Rani, R.K.R.: A Comparative Study of SaaS, PaaS and IaaS in Cloud Computing. Int. J. of Adv. Research in Computer Science and Software Engineering, pp. 458-461 (2014)

10. Serra, J.: IaaS, PaaS, and SaaS explained. James Serra's Blog (2014)

11. IaaS, PaaS and SaaS Terms Clearly Explained and Defined, http://www.silverlighthack.com/post/2011/02/27/IaaS-PaaS-and-SaaS-Terms-Explainedand-Defined.aspx

12. Tsidulko, J.: Pole Position, Ranking The Top 5 IaaS, PaaS and Private Cloud Providers. http://www.crn.com/slide-shows/cloud/300076702/pole-position-ranking-the-top-5-iaaspaas-and-private-cloud-providers.htm (2015) 
13. Badidi, E.: A Framework for Software-as-a-Service Selection and Provisioning. International Journal of Computer Networks \& Communications (IJCNC), pp. 189-200 (2013)

14. Banerjee, S., Gupta. N., Gupta, V.: Implementation and Management of framework for PaaS in Cloud Computing. International Journal of Innovations \& Advancement in Computer Science, pp. 38-49 (2014)

15. Zhang, M., Ranjan, R., Nepal, S., Menzel, M., Haller, A.: A Declarative Recommender System for Cloud Infrastructure Services Selection. In: $9^{\text {th }}$ International Conference on Economics of Grids, Clouds, Systems and Services (2012)

16. Papaioannou, I.V., Tsesmetzis, D.T., Roussaki, I.G., Anagnostou, M.E.: A QoS ontology language for Web-services. In: 20th Int. Conference on Advanced Information Networking and Applications (2006)

17. S.K.G.e.: SMICloud: A framework for comparing and ranking cloud services. In: Fourth IEEE International Conference on Utility and Cloud Computing (2011)

18. Tahir, Z., Rabbani, M., Muhammad, A., Martinez-Enriquez, A. M.: Cloud Service Recommender System Using Clustering. In: 11th International Conference on Electrical Engineering, Computing Science and Automatic Control (CCE), IEEE (2014)

19. Gang, J.W., Keqing, H., Weidong, Z., Panpan, G.: Cold-Start Web Service Recommendation Using Implicit Feedback. In: SEKE, pp. 371-376 (2014)

20. Koren, Y.: Factorization Meets the Neighborhood: A Multifaceted Collaborative Filtering Model. In: International Conference on Knowledge Discovery and Data Mining, pp. 426434 (2008)

21. Al-Moayed, A., Bernhard, H.: Quality of Service Attributes in Web Services. In: $5^{\text {th }}$ International Conference on Software Engineering Advances, pp. 367-372 (2010)

22. O'Brien, L., Merson, P., Bass, L.: Quality Attributes for Service-Oriented Architectures. In: SDSOA Proceedings of the International Workshop on Systems Development in SOA Environments (2007) 